\title{
MICRO-POWER WIRELESS TRANSMITTER WITH PRINTED BATTERY FOR MEMS SENSING AND COMMUNICATION APPLICATIONS
}

\author{
Michael Suster, Darrin J. Young, and Wen H. Ko \\ Department of EECS, Case Western Reserve University \\ Cleveland, Ohio 44106
}

\begin{abstract}
A micro-power tunnel-diode-based LC-tuned oscillator architecture is proposed for MEMS sensing and wireless data transmission applications. The prototype sensing and transmitting module employs a MEMS capacitive pressure sensor performing pressure to frequency conversion and a miniature on-board loop serving as the inductor for the LC tank and also a transmitting antenna. The system achieves a reliable telemetry performance over a distance of three meters with a total power consumption of $65 \mu \mathrm{W}$. Printed zinc-air batteries have been demonstrated as a power supply for the prototype system. The battery provides an output voltage around $1.4 \mathrm{~V}$ and can sustain a continuous telemetry operation over five hours.
\end{abstract}

\section{INTRODUCTION}

Low power miniature wireless sensor communication network is critical for biomedical implants and various industrial applications. A number of wireless modules have been developed to provide data telemetry capabilities for MEMS sensors used for biomedical implants $[1,2,3,4,5$, $6,7,8]$. In these applications, sensors such as pressure sensors, strain gauge transducers, etc. are interfaced with active electronic circuits that convert the sensing information to frequency $[3,8]$ or to voltage $[4,5,6,7]$, which is further digitized before wireless transmission. Passive RF powering and telemetry schemes are commonly implemented with inductive coupling coils, which, however, result in a limited telemetry distance on the order of several inches $[1,3,4,5,8,9]$. Active transmitters employing $\mathrm{RF}$ oscillators achieve an enhanced telemetry distance but with the penalty of excessive power dissipation [7]. Therefore, it is highly desirable to develop and demonstrate low power miniature sensing and data transmission systems achieving an enhanced telemetry distance. In this paper, we report a 65 $\mu \mathrm{W}$ wireless sensor communication module achieving a telemetry distance of three meters, suitable for various biomedical and industrial applications.

\section{MEMS SENSOR AND TRANSMIITER}

Figure 1 presents the proposed architecture, which consists of a tunnel-diode-based LC-tuned oscillator transmitter employing a MEMS capacitive pressure sensor interfaced with an on-board loop inductor also functioning as a transmission antenna, attractive for system implementation. The negative resistance of the tunnel diode under a proper bias condition compensates the tank loss, thus developing an oscillation. The oscillator output frequency is determined by the LC tank resonance. The MEMS capacitive pressure sensor thus converts the pressure information to a capacitance change resulting in the oscillator output frequency variation, which can be detected by an external receiver. This pressure to frequency modulation scheme is attractive for achieving a reliable data transmission compared to other amplitude modulation techniques.

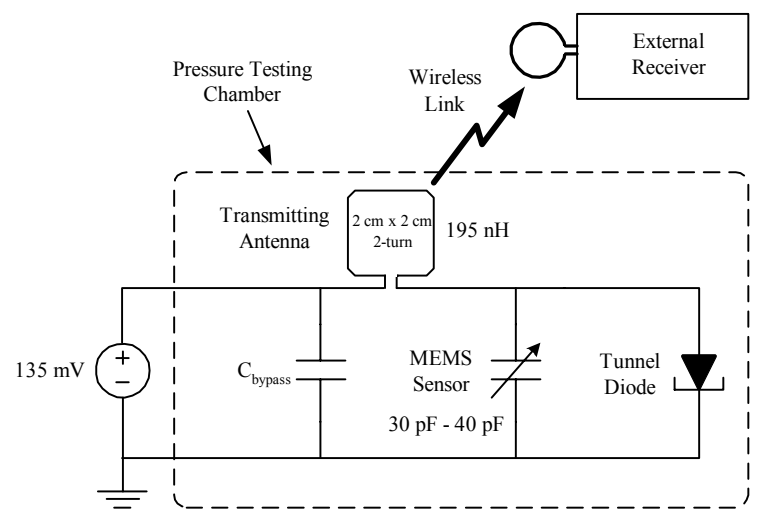

Figure 1. Wireless Transmitter Architecture

Figure 2 shows a typical I-V characteristic of a commercial germanium tunnel diode employed in the current prototype design.

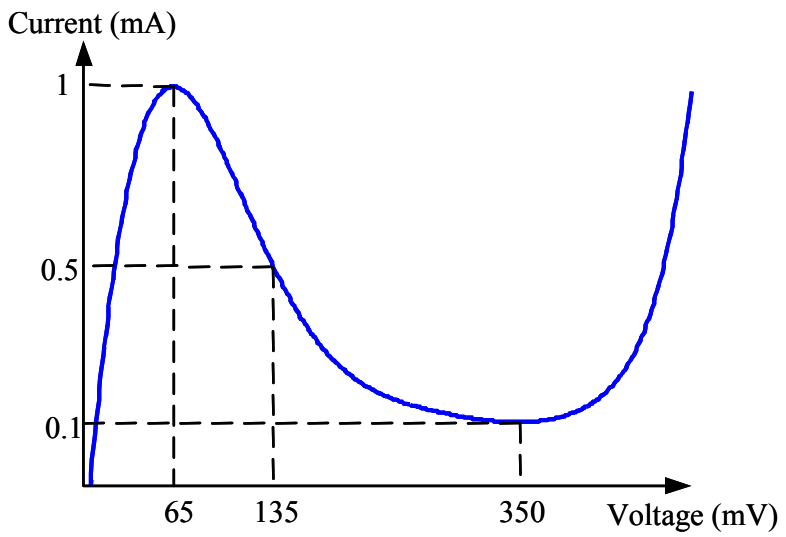

Figure 2. Tunnel Diode I-V Curve

A DC supply voltage around $135 \mathrm{mV}$ is typically needed to bias the diode thus significantly minimizing the power dissipation, a key advantage over other conventional electronic oscillator designs. The device consumes an 
average DC current of $0.5 \mathrm{~mA}$, hence $65 \mu \mathrm{W}$ power dissipation. A reduced power consumption is expected through using small-current diodes. Silicon tunnel diodes can also be used for implementing the prototype system and are attractive for potential integration with silicon-based MEMS pressure sensors, achieving further system miniaturization.

Figure 3 shows a photo of the MEMS sensor and transmitter prototype system.

Two-Turn, $2 \mathrm{~cm}$ × $2 \mathrm{~cm}$
Loop Inductor/Antenna

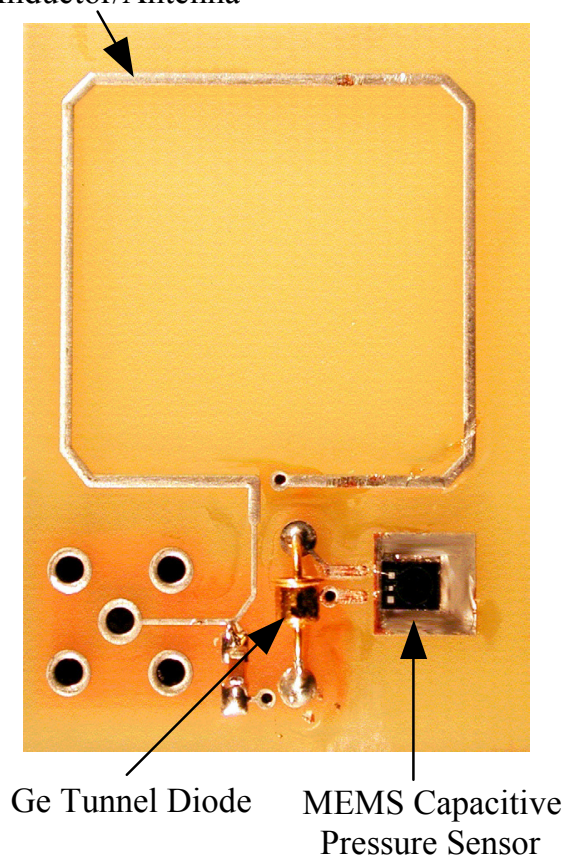

Figure 3. Photo of Prototype System

It consists of a MEMS pressure sensor interfaced with a discrete germanium tunnel diode and an on-board square loop inductor through wire bonding. A MEMS touch-mode capacitive pressure sensor [10] is employed as a demonstration vehicle for the current prototype. Figure 4 presents a simplified cross-section view of the device with detailed fabrication process outlined in [10].

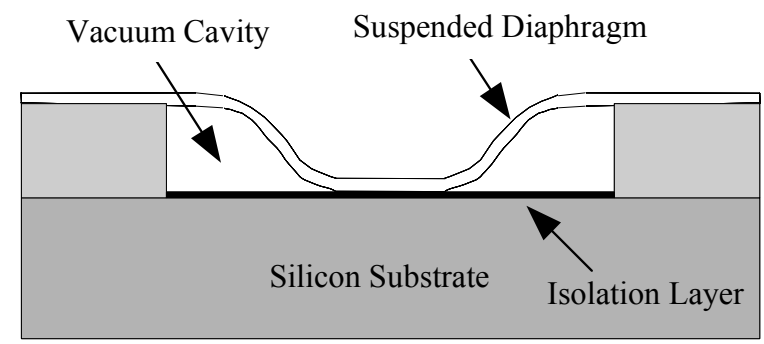

Figure 4. Sensor Cross-Section View

The sensor consists of an edge-clamped silicon diaphragm over a vacuum cavity. The diaphragm deflects under an increasing external pressure and touches the substrate causing a linear increase in sensor capacitance value beyond the touch point pressure. Figure 5 shows a typical device characteristic response between the capacitance value and applied pressure.

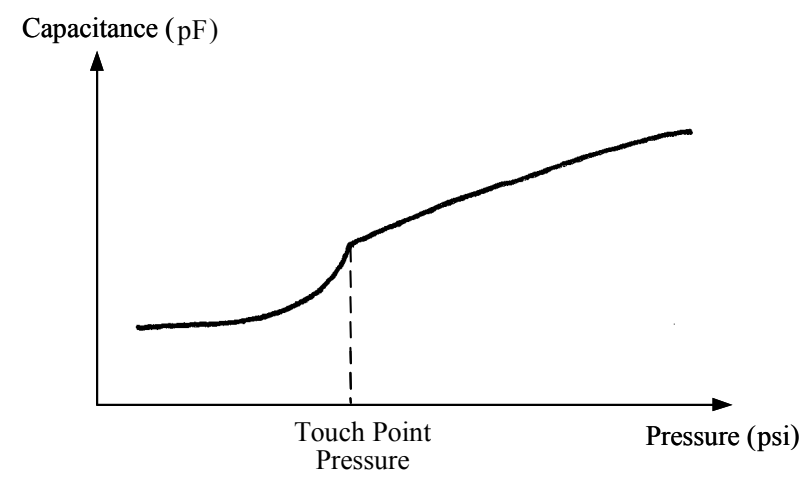

Figure 5. MEMS Pressure Sensor Characteristic

A MEMS pressure sensor consisting of a circular diaphragm with a diameter of $0.8 \mathrm{~mm}$, shown in Figure 6, exhibits a touch point pressure of 8 psi and capacitance values ranging from $30 \mathrm{pF}$ at $2 \mathrm{psi}$ to $40 \mathrm{pF}$ at $32 \mathrm{psi}$ (absolute pressures), a useful range for biomedical applications.

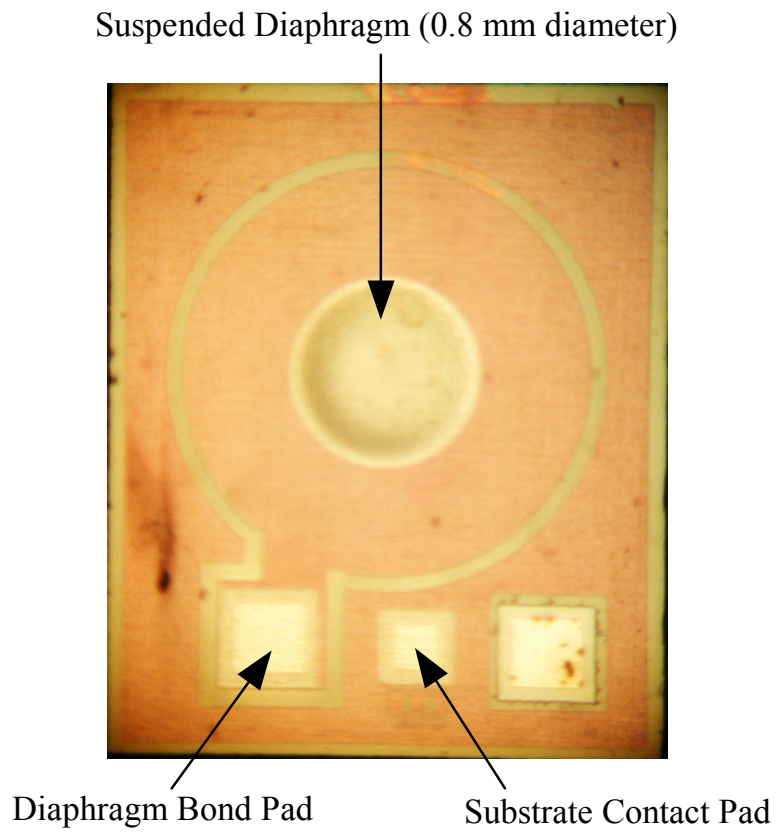

Figure 6. MEMS Pressure Sensor

This device is interfaced with a two-turn, $2 \mathrm{~cm} \times 2 \mathrm{~cm}$ onboard square loop providing $195 \mathrm{nH}$ inductance value to form the LC tank circuit as illustrated in Figures 1 and 3. The loop is also used as a transmission antenna for data telemetry. The current loop size is constrained by the operating frequency around $50 \mathrm{MHz}$, limited by the large resistive loss of the pressure sensor. Increased operating frequencies can be achieved with redesigned high-Q capacitive sensors thus reducing the loop dimension, attractive for system miniaturization. High-Q capacitive 
sensors and loop inductors are also critical for minimizing bias current required for tunnel diodes, crucial for low power applications.

\section{EXPERIMENT RESULTS}

Figure 7 shows the experiment setup for the wireless sensor communication module.

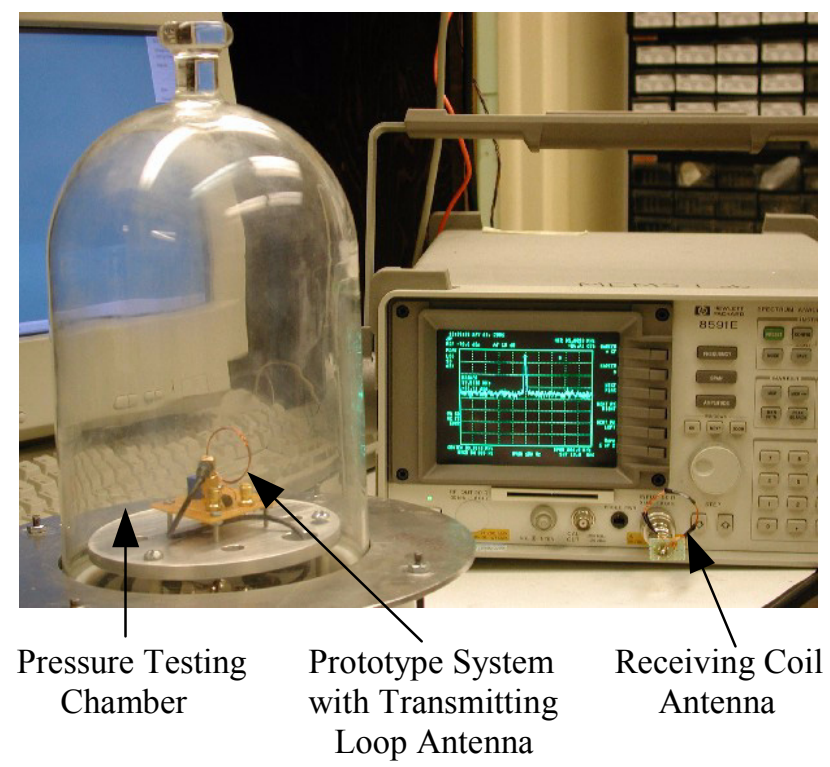

Figure 7. Experiment Setup

The prototype system is positioned inside a pressure test chamber. Wound wire coil loops were used for the early designs and are shown here for illustration purpose. A spectrum analyzer is used as an external receiver with a receiving loop antenna, similar to the transmission one, tuned to the nominal operating frequency and connected to the input port through a buffer. The oscillator operates around $53.5 \mathrm{MHz}$ at 1 atm. Its frequency varies from 55.3 $\mathrm{MHz}$ to $51.1 \mathrm{MHz}$ through pressure increase from 3 psi to 33 psi (absolute pressures), as shown in Figure 8.

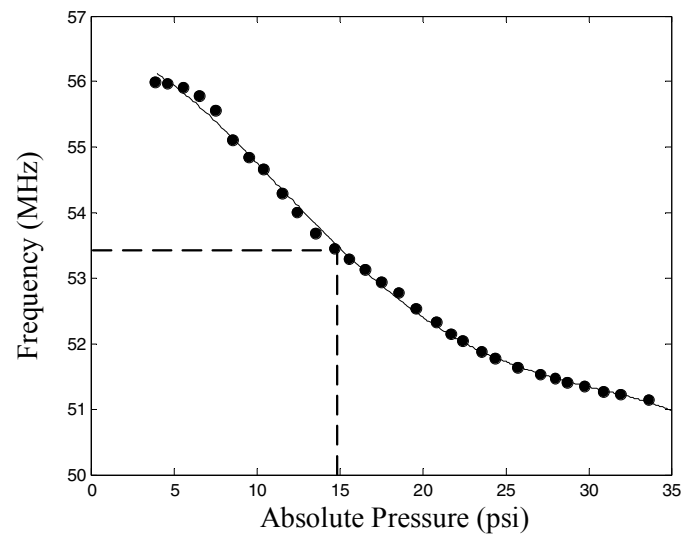

Figure 8. Oscillator Frequency Versus Pressure
Figure 9 presents the received power versus telemetry distance under $1 \mathrm{~atm}$, indicating that the spectrum analyzer can receive incoming signal with an SNR of at least $10 \mathrm{~dB}$ over a distance of three meters.

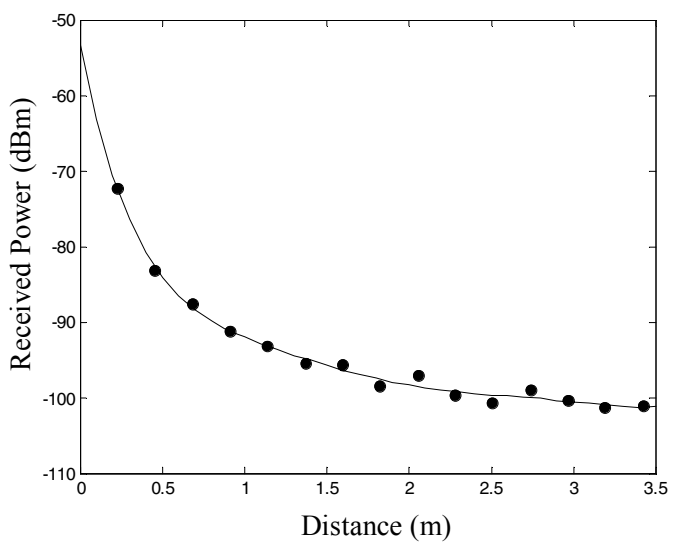

Figure 9. Received Power Versus Distance

Figure 10 shows a corresponding received power spectrum at that distance. An extended communication range is expected through using a more sensitive receiver.

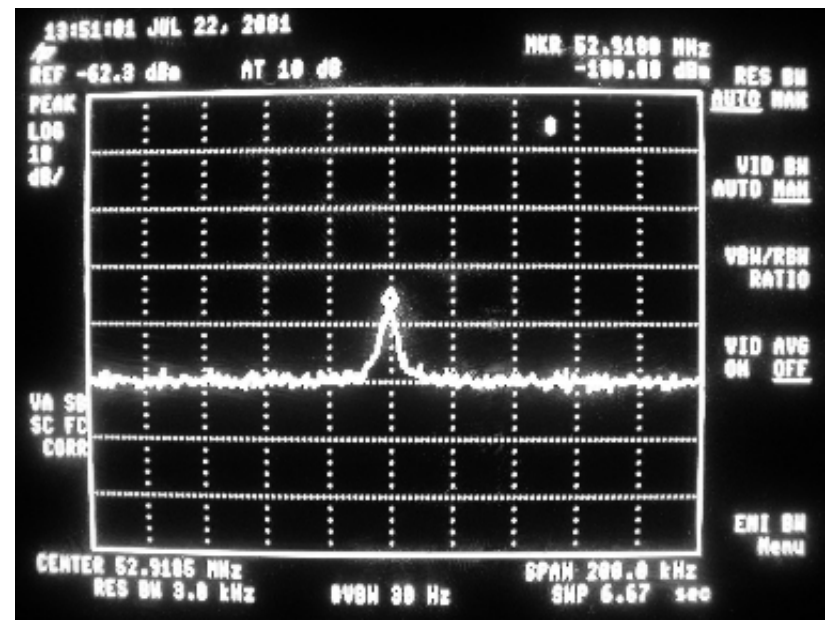

Figure 10. Received Power Spectrum

Further system miniaturization has also been explored by replacing the MEMS capacitive sensor with a high-Q fixed-value capacitor. An increased operating frequency of $80 \mathrm{MHz}$ has been obtained with a two-turn, $0.5 \mathrm{~cm} \times 0.5 \mathrm{~cm}$ on-board square loop inductor. The system achieves a telemetry distance of 0.7 meters with the same power dissipation.

A long-term frequency drift of $25 \mathrm{KHz}$ over a few days has been observed in the prototype, limiting the system resolution. An improved design employing sensor electronic interface and analog to digital conversion circuits combined with a tunnel-diode-based frequency-shift-keying (FSK) oscillator transmitter will be considered in the future to achieve a low-power high-resolution performance. 
Printed zinc-air batteries, designed and fabricated by the Chemical Engineering Department at CWRU, have been demonstrated as a power supply for the prototype system. The battery, consisting of stacked layers of electrodes and electrolyte with a dimension of approximately $2.5 \mathrm{~cm} \times 2.5$ $\mathrm{cm} \times 1 \mathrm{~mm}$, provides an output voltage around $1.4 \mathrm{~V}$ and can sustain a continuous operation of wireless module over five hours. A bias network, shown in Figure 11, is designed to properly bias the tunnel-diode oscillator transmitter [11].

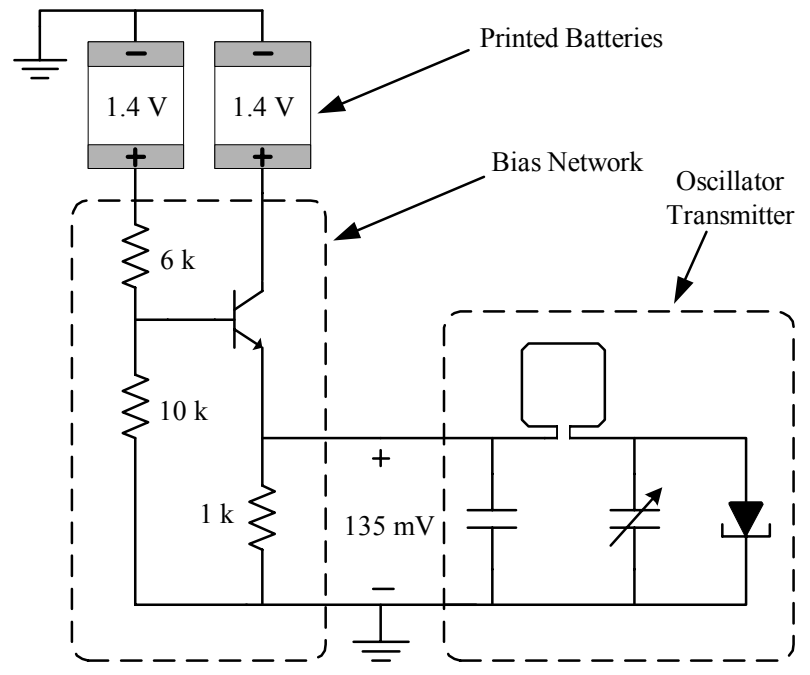

Figure 11. Oscillator Transmitter with Printed Battery Bias Network

The thick film printing process is attractive for potentially implementing the batteries on the wireless module substrate, miniaturizing overall system size. The oscillator power dissipation can be further minimized through using a low duty cycle clock to activate the transmission system for small bandwidth signals. This technique will increase the system operation time by orders of magnitude.

\section{CONCLUSION}

Tunnel-diode-based oscillator transmitter is attractive for low-power MEMS sensing and telemetry applications. The prototype wireless sensing and communication module achieves a three-meter telemetry distance with $65 \mu \mathrm{W}$ power dissipation. The proposed architecture can also serve as a low-power telemetry platform for general sensing and wireless communication applications. Printed zinc-air batteries are capable of powering the prototype design over five hours and can be potentially implemented on the wireless module substrate to achieve system miniaturization.

\section{ACKNOWLEDGMENT}

The authors would like to thank Professor Chung-Chiun Liu of the Chemical Engineering Department at Case Western Reserve University for supplying the printed zincair batteries. This work is partially supported by NASA under Glennan Microsystem Initiative. Travel support has been generously provided by the Transducers Research Foundation and by the DARPA MEMS and DARPA BioFlips programs.

\section{REFERENCES}

[1] W-H Ko and M-R Neuman, "Implant Biotelemetry and Microelectronics,” Science, Vol.15, No. 3773, pp. 351-360, April, 1967.

[2] W-H Ko, E.Yon, S. Mabrouk and J. Hynecek, "Tapedon Heart Rate and Electrocardiogram Telemetry Transmitters," J. of Asso. for Advancement of Med. Instrumentation, Vol.5, No.5, pp268-272, 1971.

[3] S. Chatzandroulis, D. Tsoukalas, and P. A. Neukomm, "A miniature Pressure System with a Capacitive Sensor and a Passive Telemetry Link for Use in Implantable Applications," Journal of MicroElectroMechanical Systems, Vol. 9, No. 1, pp. 18-23, March, 2000.

[4] D. Dudenbostel, K-L Krieger, C. Candler, and R. Laur, "A New Passive CMOS Telemetry Chip To Receive Power and Transmit Data For A Wide Range of Sensor Applications," 1997 International Conference on SolidState Sensor and Actuators, pp. 995-998, June, 1997.

[5] C. Hierold, et al, "Implantable Low Power Integrated Pressure Sensor System For Minimal Invasive Telemetric Patient Monitoring," Proceeding of MEMS 98, pp. 568-573, January, 1998.

[6] Q Huang and M. Oberle, "A 0.5-mW Passive Telemetry IC for Biomedical Applications," IEEE Journal of SolidState Circuits, Vol. 33, No. 7, pp. 937-946, July, 1998.

[7] J-B Begueret, M. R. Benbrahim, Z. Li, F. Rodes, and J-P Dom, "Converters Dedicated to Long-Term Monitoring of Strain Gauge Transducers," IEEE Journal of Solid-State Circuits, Vol. 32, No. 3, pp. 349-356, March, 1997.

[8] K. Stangel, S. Kolnsberg, D. Hammerschmidt, B. J. Hosticka, H. K. Trieu, and W. Mokwa, "A Programmable Intraocular CMOS Pressure Sensor System Implant," IEEE Journal of Solid-State Circuits, Vol. 36, No. 7, pp. 10941100, July, 2001.

[9] M. A. Fonseca, J. M. English, M. von Arx, and M. G. Allen, "High Temperature Characterization of Ceramic Pressure Sensors," Proceedings of the $11^{\text {th }}$ International Conference on Solid-State Sensors and Actuators, pp. 486489, June, 2001.

[10] W. H. Ko and Q. Wang, "Touch Mode Capacitive Pressure Sensors," Sensors and Actuators 75 (1999), pp. 242-251.

[11] W. F. Chow, Principles of Tunnel Diode Circuit, Wiley, New York, 1964. 